\title{
Two blow-up criteria of solutions to a modified two-component Camassa-Holm system
}

\author{
Caochuan $\mathrm{Ma}^{1}$, Liangbing $\mathrm{Jin}^{2^{*}}$ and Yanyi Jin²
}

"Correspondence: Ibjin@zjnu.edu.cn 2Department of Mathematics, Zhejiang Normal University, Jinhua, 321004, China

Full list of author information is available at the end of the article

\begin{abstract}
In this paper, we establish two sufficient conditions on the initial data to guarantee a blow-up phenomenon for the modified two-component Camassa-Holm (MCH2) system.

MSC: $37 \mathrm{~L} 05 ; 35 \mathrm{Q} 58 ; 26 \mathrm{~A} 12$
\end{abstract}

Keywords: $\mathrm{MCH} 2$ system; blow-up

\section{Introduction}

In this paper, we consider the Cauchy problem of the following modified two-component Camassa-Holm (MCH2) system:

$$
\begin{cases}u_{t}-u_{x x t}+3 u u_{x}-2 u_{x} u_{x x}-u u_{x x x}+g \rho \bar{\rho}_{x}=0, & t>0, x \in \mathbb{R}, \\ \rho_{t}+(\rho u)_{x}=0, & t>0, x \in \mathbb{R}, \\ y(0, x)=y_{0}(x), & x \in \mathbb{R}, \\ \rho(0, x)=\rho_{0}(x), & x \in \mathbb{R},\end{cases}
$$

where $\rho=\left(1-\partial_{x}^{2}\right)(\bar{\rho}-\hat{\rho}), u$ denotes the velocity field, $g$ is the downward constant acceleration of gravity as applied to shallow water waves, $\bar{\rho}$ is the average density, and $\hat{\rho}$ is taken to be a constant. For convenience we let $g=1$ in this paper. The MCH2 system does admit peaked solutions in the velocity and average density; we refer to Ref. [1] for details. There the authors analytically identified the steepening mechanism that allows the singular solutions to emerge from smooth spatially confined initial data. They found that wave breaking in the fluid velocity does not imply a singularity in the pointwise density $\rho$ at the point of vertical slope. Some other recent works can be found in $[2,3]$. We find that the $\mathrm{MCH} 2$ system is expressed in terms of an averaged or filtered density $\bar{\rho}$ in analogy to the relation between momentum and velocity by setting $\rho=\left(1-\partial_{x}^{2}\right)(\bar{\rho}-\hat{\rho})$. Note that the $\mathrm{MCH} 2$ system is a version of the $\mathrm{CH} 2$ system modified to allow for a dependence on the average density $\bar{\rho}$ (or depth, in the shallow water interpretation) as well as the pointwise density $\rho$.

Let $\gamma=\bar{\rho}-\hat{\rho}$, then $\gamma=G * \rho$, where the $\operatorname{sign} *$ denotes the spatial convolution, $G(x)$ is the associated Green's function of the operator $\left(1-\partial_{x}^{2}\right)^{-1}$. Therefore system (1.1) is equivalent

\section{Springer}

(02014 Ma et al.; licensee Springer. This is an Open Access article distributed under the terms of the Creative Commons Attribution License (http://creativecommons.org/licenses/by/2.0), which permits unrestricted use, distribution, and reproduction in any medium, provided the original work is properly cited. 
to the following one:

$$
\begin{cases}u_{t}+u u_{x}+\partial_{x}\left(G *\left(u^{2}+\frac{1}{2} u_{x}^{2}+\frac{1}{2} \gamma^{2}-\frac{1}{2} \gamma_{x}^{2}\right)\right)=0, & t>0, x \in \mathbb{R}, \\ \gamma_{t}+u \gamma_{x}+G *\left(\left(u_{x} \gamma_{x}\right)_{x}+u_{x} \gamma\right)=0, & t>0, x \in \mathbb{R}, \\ u(0, x)=u_{0}(x), & x \in \mathbb{R}, \\ \gamma(0, x)=\gamma_{0}(x), & x \in \mathbb{R} .\end{cases}
$$

The $\mathrm{MCH} 2$ may not be integrable unlike $\mathrm{CH} 2$. The characteristic is that it will amount to strengthening the norm for $\bar{\rho}$ from $L^{2}$ to $H^{1}$ in the potential energy term [4]. It means we have the following conserved quantity:

$$
E(t)=\int_{\mathbb{R}} u^{2}+u_{x}^{2}+\gamma^{2}+\gamma_{x}^{2} d x
$$

We cannot obtain the conservation of the $H^{1}$ norm for the $\mathrm{CH} 2$ system, which reads

$$
\begin{cases}u_{t}-u_{x x t}+3 u u_{x}-2 u_{x} u_{x x}-u u_{x x x}+g \rho \rho_{x}=0, & t>0, x \in \mathbb{R}, \\ \rho_{t}+(\rho u)_{x}=0, & t>0, x \in \mathbb{R}, \\ u(0, x)=u_{0}(x), & x \in \mathbb{R}, \\ \rho(0, x)=\rho_{0}(x), & x \in \mathbb{R} .\end{cases}
$$

The $\mathrm{CH} 2$ system appeared initially in [5], and recently Constantin and Ivanov in [6] gave a demonstration about its derivation in view of the fluid shallow water theory from the hydrodynamic point of view. This generalization, similar to the Camassa-Holm equation, possesses the peakon, multi-kink solutions and the bi-Hamiltonian structure $[7,8]$ and is integrable. Well-posedness and the wave breaking mechanism were discussed in [9-11] and the existence of global solutions was analyzed in $[6,10,12]$. The geometric investigation can be found in $[13,14]$.

Obviously, under the constraint of $\rho(x, t)=0$, this system reduces to the Camassa-Holm equation, which was derived physically by Camassa and Holm in [15] (found earlier by Fokas and Fuchssteiner [16] as a bi-Hamiltonian generalization of the KdV equation) by directly approximating the Hamiltonian for Euler's equation in the shallow water region with $u(x, t)$ representing the free surface above a flat bottom. Some satisfactory results have been obtained recently, for instance, see Refs. [17-21]. Moreover, wave breaking criteria for a large class of initial data have been established in [18, 20-22]. In [20], McKean established the necessary and sufficient condition of wave breaking, while Zhou and his collaborators gave a new and direct proof in [22] for McKean's theorem. In [23], Xin and Zhang showed global existence of weak solutions but uniqueness was obtained only under a priori assumption that is known to hold only for initial data $u_{0}(x) \in H^{1}$ such that $u_{0}(x)-u_{0 x x}(x)$ is a sign-definite random measure. The solitary waves of the CamassaHolm equation are peaked solutions and are orbitally stable [24]; see also [25] for a very related rod equation. Recently, an asymptotic analysis was given in [26]. If $\rho(x, t) \neq 0$, the $\mathrm{CH} 2$ system which includes both velocity and density variables in the dynamics is actually an extension of the $\mathrm{CH}$ equation. Although possessing peaked solutions in the velocity, the $\mathrm{CH} 2$ system does not admit singular solutions in the density profile. Its mathematical properties have been studied further in many works [6-10, 27, 28]. 
In Section 2, we recall some preliminary results on well-posedness and blow-up scenario. In Section 3, two detailed blow-up criteria are presented.

\section{Preliminaries}

In this section, for completeness, we recall some elementary results and skip their proofs. Local well-posedness for the MCH2 system can be obtained by Kato's semi-group theory [29]. In [2], the authors gave a detailed description on the well-posedness theorem.

Theorem 2.1 [2] Give $X_{0}=\left(u_{0}, \gamma_{0}\right)^{T} \in H^{s} \times H^{s-1}, s \geq 5 / 2$, there exist a maximal $T=$ $T\left(\left\|X_{0}\right\|_{H^{s} \times H^{s-1}}\right)>0$ and a unique solution $X=(u, \gamma)^{T}$ to system (1.2) such that

$$
X=X\left(\cdot, X_{0}\right) \in C\left([0, T], H^{s} \times H^{s-1}\right) \cap C^{1}\left([0, T], H^{s-1} \times H^{s-2}\right) .
$$

Moreover, the solution depends continuously on the initial data, i.e. the mapping

$$
X_{0} \rightarrow X\left(\cdot, X_{0}\right): H^{s} \times H^{s} \rightarrow C\left([0, T], H^{s} \times H^{s-1}\right) \cap C^{1}\left([0, T], H^{s-1} \times H^{s-2}\right)
$$

is continuous.

The next result describes the precise blow-up scenario for sufficiently regular solutions to system (1.2).

Theorem 2.2 [2] Let $X_{0}=\left(u_{0}, \gamma_{0}\right)^{T} \in H^{s} \times H^{s-1}, s \geq 5 / 2$, and Let $T$ be the maximal existence time of the solution $X=(u, \gamma)^{T}$ to system (1.2) with the initial data $X_{0}$. Then the corresponding solution blows up in finite time if and only if

$$
\liminf _{t \rightarrow T} u_{x \in \mathbb{R}}(x, t)=-\infty
$$

We also need to introduce the classical particle trajectory method for later use. Let $q(x, t)$ be the particle line evolved by the solution; that is, it satisfies

$$
\frac{d q(x, t)}{d t}=u(q(x, t), t), \quad q(x, t=0)=x .
$$

Differentiating the first equation with respect to $x$, one has

$$
\frac{d}{d x} q_{t}=q_{x t}=u_{x}(q, t) q_{x}, \quad t \in(0, T)
$$

Hence

$$
q_{x}(x, t)=e^{\int_{0}^{t} u_{x}(q, s) d s}, \quad q_{x}(x, 0)=1,
$$

which is always positive before the blow-up time. Therefore, the function $q(x, t)$ is an increasing diffeomorphism of the line. 


\section{Blow-up}

Before giving blow-up theorems, we rewrite the system (1.1) by $y=u-u_{x x}$ as follows:

$$
\begin{cases}y_{t}+u y_{x}+2 y u_{x}+g \rho \bar{\rho}_{x}=0, & t>0, x \in \mathbb{R}, \\ \rho_{t}+(\rho u)_{x}=0, & t>0, x \in \mathbb{R}, \\ y(0, x)=y_{0}(x), & x \in \mathbb{R}, \\ \rho(0, x)=\rho_{0}(x), & x \in \mathbb{R} .\end{cases}
$$

As $y(x, t)=\left(1-\partial_{x}^{2}\right) u(x, t)$ and as $u(x, t)$ is given by the convolution $u(x, t)=G * y$ with $G=\frac{1}{2} e^{-|x|}$, we have

$$
u(x, t)=\frac{1}{2} e^{-x} \int_{-\infty}^{x} e^{\xi} y(\xi, t) d \xi+\frac{1}{2} e^{x} \int_{x}^{\infty} e^{-\xi} y(\xi, t) d \xi,
$$

from which we get

$$
u_{x}(x, t)=-\frac{1}{2} e^{-x} \int_{-\infty}^{x} e^{\xi} y(\xi, t) d \xi+\frac{1}{2} e^{x} \int_{x}^{\infty} e^{-\xi} y(\xi, t) d \xi
$$

Thus,

$$
\begin{aligned}
& \left(u+u_{x}\right)(x, t)=e^{x} \int_{x}^{\infty} e^{-\xi} y(\xi, t) d \xi, \\
& \left(u-u_{x}\right)(x, t)=e^{-x} \int_{-\infty}^{-x} e^{\xi} y(\xi, t) d \xi .
\end{aligned}
$$

Now we give our two blow-up theorems.

Theorem 3.1 Suppose $X_{0}=\left(u_{0}, \gamma_{0}\right)^{T} \in H^{s} \times H^{s-1}, s \geq \frac{5}{2}$, for some point $x_{0} \in \mathbb{R}, \rho_{0}\left(x_{0}\right)=$ $y_{0}\left(x_{0}\right)=0$ and initial data satisfies the following conditions:

(i) $\int_{-\infty}^{x_{0}} e^{\xi} y_{0}(\xi) d \xi>0$ and $\int_{x_{0}}^{\infty} e^{-\xi} y_{0}(\xi) d \xi<0$,

(ii) $\int_{-\infty}^{x_{0}} e^{\xi} y_{0}(\xi) d \xi \int_{x_{0}}^{\infty} e^{-\xi} y_{0}(\xi) d \xi+E(0)<0$,

where $E(0)=\left\|u_{0}\right\|_{H^{1}}^{2}+\left\|\gamma_{0}\right\|_{H^{1}}^{2}$. Then the solution to system (1.2) with the initial value $X_{0}$ blows up in finite time.

Remark 3.1 In fact the condition (ii) can be reduced to

$$
\int_{-\infty}^{x_{0}} e^{\xi} y_{0}(\xi) d \xi \int_{x_{0}}^{\infty} e^{-\xi} y_{0}(\xi) d \xi+\int_{\mathbb{R}}\left(\gamma^{2}+\gamma_{\xi}^{2}\right)(\xi, t) d \xi<0
$$

If $\gamma(\xi, t) \equiv 0$, the theory becomes the blow-up theorem in [21] for the Camassa-Holm. As $\gamma(x, t)$ has nothing to do with the initial data, so we add the initial energy $E(0)$ to condition (ii).

Proof Differentiating the first equation in system (1.2) with respect to $x$, we obtain

$$
u_{t x}+u u_{x x}+u_{x}^{2}+\partial_{x}^{2}\left(G *\left(u^{2}+\frac{1}{2} u_{x}^{2}+\frac{1}{2} \gamma^{2}-\frac{1}{2} \gamma_{x}^{2}\right)\right)=0 .
$$


Applying $\partial_{x}^{2}(G * f)=G * f-f$ to the above equation yields

$$
u_{t x}+u u_{x x}=u^{2}-\frac{1}{2} u_{x}^{2}+\frac{1}{2} \gamma^{2}-\frac{1}{2} \gamma_{x}^{2}-G *\left(u^{2}+\frac{1}{2} u_{x}^{2}+\frac{1}{2} \gamma^{2}-\frac{1}{2} \gamma_{x}^{2}\right)
$$

This equation gives

$$
\begin{aligned}
\frac{d}{d t} u_{x}\left(q\left(x_{0}, t\right), t\right)= & \left(u_{x t}+u u_{x x}\right)\left(q\left(x_{0}, t\right), t\right) \\
\leq & \frac{1}{2} u^{2}\left(q\left(x_{0}, t\right), t\right)-\frac{1}{2} u_{x}^{2}\left(q\left(x_{0}, t\right), t\right) \\
& +\frac{1}{2} \gamma^{2}\left(q\left(x_{0}, t\right), t\right)-\frac{1}{2} \gamma_{x}^{2}\left(q\left(x_{0}, t\right), t\right)-\frac{1}{2} G *\left(\gamma^{2}-\gamma_{x}^{2}\right) \\
\leq & \frac{1}{2} u^{2}\left(q\left(x_{0}, t\right), t\right)-\frac{1}{2} u_{x}^{2}\left(q\left(x_{0}, t\right), t\right) \\
& +\frac{1}{2} \gamma^{2}\left(q\left(x_{0}, t\right), t\right)+\frac{1}{2} G * \gamma_{x}^{2},
\end{aligned}
$$

where we used the fact

$$
G *\left(u^{2}+\frac{1}{2} u_{x}^{2}\right) \geq \frac{1}{2} u^{2}
$$

As regards $\gamma^{2}$ we can deduce that

$$
\begin{aligned}
\gamma^{2} & =\int_{-\infty}^{x} \gamma \gamma_{x} d x-\int_{x}^{\infty} \gamma \gamma_{x} d x \leq \int_{-\infty}^{x} \frac{\gamma^{2}+\gamma_{x}^{2}}{2} d x+\int_{\infty}^{x} \frac{\gamma^{2}+\gamma_{x}^{2}}{2} d x \\
& =\int_{-\infty}^{\infty} \frac{\gamma^{2}+\gamma_{x}^{2}}{2} d x \leq \frac{1}{2} E(0) .
\end{aligned}
$$

Due to (3.2), we obtain

$$
\gamma^{2} \leq\|\gamma\|_{L^{\infty}}^{2} \leq \frac{1}{2} E(0)
$$

Owing to $G * f=\frac{1}{2} \int_{\mathbb{R}} e^{-|x-\xi|} f(\xi) d \xi$, we have the following inequality:

$$
\begin{aligned}
G * \gamma_{x}^{2} & =\frac{1}{2} \int_{\mathbb{R}} e^{-|x-\xi|} \gamma_{x}^{2}(\xi) d \xi \leq \frac{1}{2} \int_{-\infty}^{x} \gamma_{x}^{2} d \xi+\frac{1}{2} \int_{x}^{\infty} \gamma_{x}^{2} d \xi \\
& \leq \frac{1}{2}\left(\left\|u_{0}\right\|_{H^{1}}^{2}+\left\|\gamma_{0}\right\|_{H^{1}}^{2}\right)=\frac{1}{2} E(0) .
\end{aligned}
$$

Then using (3.3) and (3.4), we can turn the inequality (3.1) into

$$
\begin{aligned}
\frac{d}{d t} u_{x}\left(q\left(x_{0}, t\right), t\right) \leq & \frac{1}{2} u^{2}\left(q\left(x_{0}, t\right), t\right)-\frac{1}{2} u_{x}^{2}\left(q\left(x_{0}, t\right), t\right) \\
& +\frac{1}{2} \gamma^{2}\left(q\left(x_{0}, t\right), t\right)+\frac{1}{2} G * \gamma_{x}^{2} \\
\leq & \frac{1}{2} u^{2}\left(q\left(x_{0}, t\right), t\right)-\frac{1}{2} u_{x}^{2}\left(q\left(x_{0}, t\right), t\right)+\frac{1}{2} E(0)
\end{aligned}
$$

In order to reach our result, we need the following claim. 
Claim $1 u_{x}\left(q\left(x_{0}, t\right), t\right)<0$ is decreasing, $u^{2}\left(q\left(x_{0}, t\right), t\right)-u_{x}^{2}\left(q\left(x_{0}, t\right), t\right)+E(0)<0$ for all $t \in[0, T)$, where $T$ is the maximal existence time of the solution.

Suppose not, i.e., there exists a $t_{0}$ such that $u^{2}\left(q\left(x_{0}, t\right), t\right)-u_{x}^{2}\left(q\left(x_{0}, t\right), t\right)+E(0)<0$ on $\left[0, t_{0}\right)$ and $u^{2}\left(q\left(x_{0}, t_{0}\right), t_{0}\right)-u_{x}^{2}\left(q\left(x_{0}, t_{0}\right), t_{0}\right)+E(0)=0$. Now let

$$
I(t):=\frac{1}{2} e^{-q\left(x_{0}, t\right)} \int_{-\infty}^{q\left(x_{0}, t\right)} e^{\xi} y(\xi, t) d \xi
$$

and

$$
I I(t):=\frac{1}{2} e^{q\left(x_{0}, t\right)} \int_{q\left(x_{0}, t\right)}^{\infty} e^{-\xi} y(\xi, t) d \xi .
$$

Firstly for $t \in\left[0, t_{0}\right)$, differentiating $I(t)$, we have

$$
\begin{aligned}
\frac{d I(t)}{d t}= & -\frac{1}{2} u\left(q\left(x_{0}, t\right), t\right) e^{-q\left(x_{0}, t\right)} \int_{-\infty}^{q\left(x_{0}, t\right)} e^{\xi} y(\xi, t) d \xi+\frac{1}{2} e^{-q\left(x_{0}, t\right)} \int_{-\infty}^{q\left(x_{0}, t\right)} e^{\xi} y_{t}(\xi, t) d \xi \\
= & \frac{1}{2} u\left(u_{x}-u\right)\left(q\left(x_{0}, t\right), t\right)-\frac{1}{2} e^{-q\left(x_{0}, t\right)} \int_{-\infty}^{q\left(x_{0}, t\right)} e^{\xi}\left(u y_{x}+2 u_{x} y+\rho \gamma_{x}\right) d \xi \\
\geq & \frac{1}{2} u\left(u_{x}-u\right)\left(q\left(x_{0}, t\right), t\right)+\frac{1}{4}\left(u^{2}+u_{x}^{2}-2 u u_{x}\right)\left(q\left(x_{0}, t\right), t\right) \\
& -\frac{1}{4} \gamma^{2}\left(q\left(x_{0}, t\right), t\right)+\frac{1}{4} \gamma_{x}^{2}\left(q\left(x_{0}, t\right), t\right)+\frac{1}{4} G *\left(\gamma^{2}-\gamma_{x}^{2}\right) \\
\geq & \frac{1}{4}\left(u_{x}^{2}-u^{2}\right)\left(q\left(x_{0}, t\right), t\right)-\frac{1}{4} \gamma^{2}\left(q\left(x_{0}, t\right), t\right)+\frac{1}{4} \gamma_{x}^{2}\left(q\left(x_{0}, t\right), t\right) \\
& +\frac{1}{4} G *\left(\gamma^{2}-\gamma_{x}^{2}\right) \\
\geq & \frac{1}{4} u_{x}^{2}\left(q\left(x_{0}, t\right), t\right)-\frac{1}{4} u^{2}\left(q\left(x_{0}, t\right), t\right)-\frac{1}{4} \gamma^{2}\left(q\left(x_{0}, t\right), t\right)-\frac{1}{4} G * \gamma_{x}^{2} \\
\geq & \frac{1}{4}\left(u_{x}^{2}\left(q\left(x_{0}, t\right), t\right)-u^{2}\left(q\left(x_{0}, t\right), t\right)-E(0)\right)>0,
\end{aligned}
$$

where we used (3.3) and (3.4).

Secondly, by the same argument, we get

$$
\begin{aligned}
\frac{d I I(t)}{d t} \leq & -\frac{1}{4}\left(u_{x}^{2}-u^{2}\right)\left(q\left(x_{0}, t\right), t\right)+\frac{1}{4} \gamma^{2}\left(q\left(x_{0}, t\right), t\right)-\frac{1}{4} \gamma_{x}^{2}\left(q\left(x_{0}, t\right), t\right) \\
& -\frac{1}{4} G *\left(\gamma^{2}-\gamma_{x}^{2}\right) \\
\leq & -\frac{1}{4} u_{x}^{2}\left(q\left(x_{0}, t\right), t\right)+\frac{1}{4} u^{2}\left(q\left(x_{0}, t\right), t\right)+\frac{1}{4} \gamma^{2}\left(q\left(x_{0}, t\right), t\right)+\frac{1}{4} G * \gamma_{x}^{2} \\
\leq & -\frac{1}{4}\left(u_{x}^{2}\left(q\left(x_{0}, t\right), t\right)-u^{2}\left(q\left(x_{0}, t\right), t\right)-E(0)\right)<0 .
\end{aligned}
$$

Hence, it follows from (3.6) and (3.7) and the continuity property of the ODEs that

$$
\begin{aligned}
u_{x}^{2}\left(q\left(x_{0}, t\right), t\right)-u^{2}\left(q\left(x_{0}, t\right), t\right)-E(0) & =-4 I(t) I I(t)-E(0) \\
& >-4 I(0) I I(0)-E(0)>0,
\end{aligned}
$$


for all $t \in\left[0, t_{0}\right)$, where we have used the condition (i) and (ii). The continuity property implies that, when $t=t_{0}$, we have

$$
u_{x}^{2}\left(q\left(x_{0}, t_{0}\right), t_{0}\right)-u^{2}\left(q\left(x_{0}, t_{0}\right), t_{0}\right)-E(0)>0 .
$$

This is an obvious contradiction. Then $t_{0}$ can be extended to $T$. On the other hand

$$
\begin{aligned}
u_{x}\left(q\left(x_{0}, t\right), t\right) & =-\frac{1}{2} e^{-q\left(x_{0}, t\right)} \int_{-\infty}^{q\left(x_{0}, t\right)} e^{\xi} y(\xi, t) d \xi+\frac{1}{2} e^{q\left(x_{0}, t\right)} \int_{q\left(x_{0}, t\right)}^{\infty} e^{-\xi} y(\xi, t) d \xi \\
& <-\frac{1}{2} e^{-x_{0}} \int_{-\infty}^{x_{0}} e^{\xi} y_{0}(\xi, t) d \xi+\frac{1}{2} e^{x_{0}} \int_{x_{0}}^{\infty} e^{-\xi} y_{0}(\xi, t) d \xi .
\end{aligned}
$$

Then the initial assumption makes $u_{x}\left(q\left(x_{0}, t\right), t\right)<0$ obvious. So our claim is proved.

Using (3.6) and (3.7) again, we have the following equation for $\left(u_{x}^{2}-u^{2}\right)\left(q\left(x_{0}, t\right), t\right)$ :

$$
\begin{aligned}
\frac{d}{d t}\left(u_{x}^{2}-u^{2}\right)\left(q\left(x_{0}, t\right), t\right)= & -4 I I(t) \frac{d}{d t} I(t)-4 I(t) \frac{d}{d t} I I(t) \\
\geq & -I I(t)\left(u_{x}^{2}\left(q\left(x_{0}, t\right), t\right)-u^{2}\left(q\left(x_{0}, t\right), t\right)-E(0)\right) \\
& +I(t)\left(u_{x}^{2}\left(q\left(x_{0}, t\right), t\right)-u^{2}\left(q\left(x_{0}, t\right), t\right)-E(0)\right) \\
= & -u_{x}\left(q\left(x_{0}, t\right), t\right)\left(u_{x}^{2}-u^{2}-E(0)\right)\left(q\left(x_{0}, t\right), t\right),
\end{aligned}
$$

where we used $u_{x}\left(q\left(x_{0}, t\right), t\right)=-I(t)+I I(t)$. Due to (3.5), we can obtain

$$
u_{x}\left(q\left(x_{0}, t\right), t\right) \leq \int_{0}^{t} \frac{1}{2}\left(u^{2}-u_{x}^{2}+E(0)\right)\left(q\left(x_{0}, \tau\right), \tau\right) d \tau+u_{0 x}\left(x_{0}\right) .
$$

Now, substituting (3.9) into (3.8), it yields

$$
\begin{aligned}
& \frac{d}{d t}\left(u_{x}^{2}-u^{2}\right)\left(q\left(x_{0}, t\right), t\right) \\
& \geq\left(-\int_{0}^{t} \frac{1}{2}\left(u^{2}-u_{x}^{2}+E(0)\right)\left(q\left(x_{0}, \tau\right), \tau\right) d \tau-u_{0 x}\left(x_{0}\right)\right) \\
& \quad \times\left(u_{x}^{2}\left(q\left(x_{0}, t\right), t\right)-u^{2}\left(q\left(x_{0}, t\right), t\right)-E(0)\right) \\
& =\frac{1}{2}\left(\int_{0}^{t}\left(u_{x}^{2}-u^{2}-E(0)\right)\left(q\left(x_{0}, \tau\right), \tau\right) d \tau-2 u_{0 x}\left(x_{0}\right)\right) \\
& \quad \times\left(u_{x}^{2}\left(q\left(x_{0}, t\right), t\right)-u^{2}\left(q\left(x_{0}, t\right), t\right)-E(0)\right) .
\end{aligned}
$$

Before completing the proof, we need the following technical lemma.

Lemma 3.1 [30] Suppose that $\Psi(t)$ is a twice continuously differential satisfying

$$
\begin{cases}\Psi^{\prime \prime}(t) \geq C_{0} \Psi^{\prime}(t) \Psi(t), & t>0, C_{0}>0, \\ \Psi(0)>0, & \psi^{\prime}(0)>0 .\end{cases}
$$




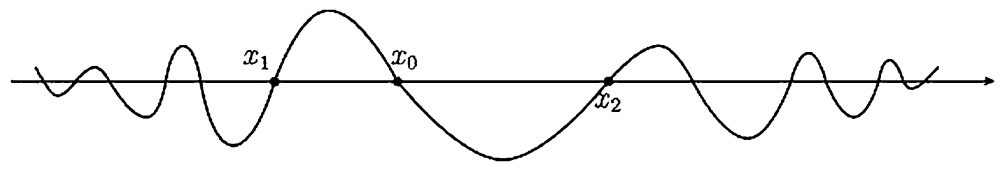

Figure $1 y_{0}$.

Then $\Psi(t)$ blows up in finite time. Moreover the blow-up time $T$ can be estimated in terms of the initial datum as

$$
T \leq \max \left\{\frac{2}{C_{0} \Psi(0)}, \frac{\Psi(0)}{\Psi^{\prime}(0)}\right\}
$$

Let $\Psi(t)=\int_{0}^{t}\left(u_{x}^{2}-u^{2}-E(0)\right)\left(q\left(x_{0}, \tau\right), \tau\right) d \tau-2 u_{0 x}\left(x_{0}\right)$, then given the condition (i) and due to the claim and the expression of $u_{0 x}\left(x_{0}\right)$, we get $\Psi(t)>0$ and $\Psi^{\prime}(t)>0$. Using the above lemma, (3.10) is an equation of type (3.11) with $C_{0}=\frac{1}{2}$. We can conclude that under the conditions (i) and (ii), the solution to system (1.2) blows up in finite time.

Theorem 3.2 Suppose $X_{0}=\left(u_{0}, \rho_{0}\right)^{T} \in H^{s} \times H^{s-1}, s \geq \frac{5}{2}$, there exists $\delta$ satisfying when $x \in\left(x_{0}-\delta, x_{0}+\delta\right), \rho_{0}(x) \equiv 0$, when $x \in\left(-\infty, x_{0}-\delta\right], \rho_{0}(x) \geq 0$ and when $x \in\left[x_{0}+\delta, \infty\right)$, $\rho_{0}(x) \leq 0$. Some portion of the positive part of $y_{0}(x)$ lies to the left of some portion of its negative part with the changing sign point at $x_{0}$, then the solution to system (1.2) with the initial value $X_{0}$ blows up in finite time.

Before we prove the above theorem, we draw a picture of $y_{0}$ in Figure 1.

Proof In order to prove the theorem, we define the following quantities:

$$
A_{0}=\int_{-\infty}^{x_{0}} e^{\xi} y_{0}(\xi) d \xi, \quad B_{0}=\int_{x_{0}}^{\infty} e^{-\xi} y_{0}(\xi) d \xi
$$

Then concerning the sign of $A_{0}$ and $B_{0}$, we have four cases.

Case 1: $A_{0}>0, B_{0}<0$.

Case 2: $A_{0}<0, B_{0}<0$.

Case 3: $A_{0}>0, B_{0}>0$.

Case 4: $A_{0}<0, B_{0}>0$.

The cases for $A_{0}=0$ or $B_{0}=0$ are easy to handle.

First, we can find that Case 3 is equivalent to Case 2.

In fact, if $(u(x, t), \gamma(x, t))$ is a solution, let $\tilde{u}(x, t)=-u(-x, t)$ and $\tilde{\gamma}(x, t)=-\gamma(-x, t)$, then $(\tilde{u}(x, t), \tilde{\gamma}(x, t))$ is also a solution with $\tilde{u_{0}}(x)=-u_{0}(-x)$ and $\tilde{\gamma_{0}}(x)=-\gamma_{0}(-x)$. Let $\tilde{y}_{0}(x)=(1-$ $\left.\partial_{x}^{2}\right) \tilde{u}_{0}(x)=-y_{0}(-x)$ with positive part on $\left(-x_{2},-x_{0}\right)$ and negative part on $\left(-x_{0},-x_{1}\right)$, then we have

$$
\tilde{A_{0}}=\int_{-\infty}^{-x_{0}} e^{\xi} \tilde{y_{0}}(\xi) d \xi=-\int_{-\infty}^{-x_{0}} e^{\xi} y_{0}(-\xi) d \xi=-\int_{x_{0}}^{\infty} e^{-\eta} y_{0}(\eta) d \eta=-B_{0}
$$

By the same reasoning, we have $\tilde{B_{0}}=-A_{0}$. 
Set

$$
A(q(x, t), t):=\int_{-\infty}^{q(x, t)} e^{\xi} y(\xi, t) d \xi \quad \text { and } \quad B(q(x, t), t):=\int_{q(x, t)}^{\infty} e^{-\xi} y(\xi, t) d \xi
$$

for any $x \in\left(x_{0}-\delta, x_{0}+\delta\right)$, then we have

$$
\begin{aligned}
\frac{d A(q(x, t), t)}{d t}= & \frac{1}{2} e^{q(x, t)}\left[u(q(x, t), t)-u_{x}(q(x, t), t)\right]^{2} \\
& +\frac{1}{2} \int_{-\infty}^{q(x, t)} e^{\xi}\left[u(\xi, t)-u_{\xi}(\xi, t)\right]^{2} d \xi-\frac{1}{2} e^{q(x, t)}\left(\gamma^{2}-\gamma_{x}^{2}\right)(q(x, t), t) \\
& +\frac{1}{2} \int_{-\infty}^{q(x, t)} e^{\xi}\left(\gamma^{2}-\gamma_{x}^{2}\right)(\xi, t) d \xi, \\
\frac{d B(q(x, t), t)}{d t}= & -\frac{1}{2} e^{-q(x, t)}\left[u(q(x, t), t)+u_{x}(q(x, t), t)\right]^{2} \\
& -\frac{1}{2} \int_{q(x, t)}^{\infty} e^{-\xi}\left[u(\xi, t)+u_{\xi}(\xi, t)\right]^{2} d \xi+\frac{1}{2} e^{-q(x, t)}\left(\gamma^{2}-\gamma_{x}^{2}\right)(q(x, t), t) \\
& -\frac{1}{2} \int_{q(x, t)}^{\infty} e^{-\xi}\left(\gamma^{2}-\gamma_{x}^{2}\right)(\xi, t) d \xi .
\end{aligned}
$$

In order to get the monotonous property of $A(q(x, t), t)$ and $B(q(x, t), t)$, we need the following claim.

Claim 2 Under the condition of $\rho_{0}(x)$ from the theorem, for all $t>0$ we have

$$
\begin{cases}\rho(q(x, t), t) \geq 0, & x \in\left(-\infty, x_{0}-\delta\right] \\ \rho(q(x, t), t) \equiv 0, & x \in\left(x_{0}-\delta, x_{0}+\delta\right) \\ \rho(q(x, t), t) \leq 0, & x \in\left[x_{0}+\delta, \infty\right)\end{cases}
$$

and $y\left(q\left(x_{0}, t\right), t\right)=0$.

From the first equation of system (1.1) we have the following equivalent form:

$$
y_{t}+2 y u_{x}+y_{x} u+\rho \gamma_{x}=0 \text {. }
$$

Applying the particle trajectory method and the second equation in (1.1), we obtain

$$
\begin{aligned}
\frac{d}{d t}\left(y(q(x, t), t) q_{x}^{2}(x, t)\right) & =\left(y_{t}+2 y u_{x}+y_{x} u\right)(q(x, t), t) q_{x}^{2}(x, t) \\
& =-\rho(q(x, t), t) \gamma_{x}(q(x, t), t) q_{x}^{2}(x, t)
\end{aligned}
$$

and

$$
\frac{d}{d t} \rho(q(x, t), t) q_{x}(x, t)=0
$$

which implies

$$
\rho(q(x, t), t) q_{x}(x, t)=\rho_{0}(x) .
$$


Due to the condition of $\rho_{0}(x)$ from the theorem and $q_{x}(x, t)>0$, we get

$$
\begin{cases}\rho(q(x, t), t) \geq 0, & x \in\left(-\infty, x_{0}-\delta\right], \\ \rho(q(x, t), t) \equiv 0, & x \in\left(x_{0}-\delta, x_{0}+\delta\right), \\ \rho(q(x, t), t) \leq 0, & x \in\left[x_{0}+\delta, \infty\right),\end{cases}
$$

for all $t>0$. Then

$$
\frac{d}{d t} y(q(x, t), t) q_{x}^{2}(x, t)=0 \quad x \in\left(x_{0}-\delta, x_{0}+\delta\right) .
$$

Thus $y(q(x, t), t) q_{x}^{2}(x, t)$ is independent on time $t$. By taking $t=0$, we have

$$
y(q(x, t), t) q_{x}^{2}(x, t)=y_{0}(x) \quad x \in\left(x_{0}-\delta, x_{0}+\delta\right) .
$$

Since $y_{0}\left(x_{0}\right)=0$ and from the above equation, we get $y\left(q\left(x_{0}, t\right), t\right)=0$. Therefore the claim holds.

Claim 3 For any fixed $t, \gamma_{x}^{2}(x, t)-\gamma^{2}(x, t) \leq\left(\gamma_{x}^{2}-\gamma^{2}\right)(q(\eta, t), t)$, for any $\eta \in\left(x_{0}-\delta, x_{0}+\delta\right)$ and all $x \in \mathbb{R}$.

As $\gamma=G * \rho$, where $G$ is the Green's function, it can be expressed as $G(x)=-\frac{1}{2} e^{-|x|}$, and then one has the equation for $\gamma(x, t)$ and $\gamma_{x}(x, t)$ :

$$
\begin{aligned}
& \gamma(x, t)=\frac{1}{2} e^{-x} \int_{-\infty}^{x} e^{\xi} \rho(\xi, t) d \xi+\frac{1}{2} e^{x} \int_{x}^{\infty} e^{-\xi} \rho(\xi, t) d \xi, \\
& \gamma_{x}(x, t)=-\frac{1}{2} e^{-x} \int_{-\infty}^{x} e^{\xi} \rho(\xi, t) d \xi+\frac{1}{2} e^{x} \int_{x}^{\infty} e^{-\xi} \rho(\xi, t) d \xi .
\end{aligned}
$$

Therefore,

$$
\begin{aligned}
& \left(\gamma+\gamma_{x}\right)(x, t)=e^{x} \int_{x}^{\infty} e^{-\xi} \rho(\xi, t) d \xi \\
& \left(\gamma-\gamma_{x}\right)(x, t)=e^{-x} \int_{-\infty}^{-x} e^{\xi} \rho(\xi, t) d \xi .
\end{aligned}
$$

By direct computation, if $x \leq q(\eta, t)$, for any $\eta \in\left(x_{0}-\delta, x_{0}+\delta\right)$, then from the above two equations we can get

$$
\begin{aligned}
\gamma_{x}^{2}(x, t)-\gamma^{2}(x, t)= & -\left(\int_{-\infty}^{q(\eta, t)} e^{\xi} \rho(\xi, t) d \xi-\int_{x}^{q(\eta, t)} e^{\xi} \rho(\xi, t) d \xi\right) \\
& \times\left(\int_{q(\eta, t)}^{\infty} e^{-\xi} \rho(\xi, t) d \xi+\int_{x}^{q(\eta, t)} e^{-\xi} \rho(\xi, t) d \xi\right) \\
= & \left(\gamma_{x}^{2}-\gamma^{2}\right)(q(\eta, t), t)-\int_{-\infty}^{q(\eta, t)} e^{\xi} \rho(\xi, t) d \xi \int_{x}^{q(\eta, t)} e^{-\xi} \rho(\xi, t) d \xi \\
& +\int_{x}^{q(\eta, t)} e^{\xi} \rho(\xi, t) d \xi \int_{q(\eta, t)}^{\infty} e^{-\xi} \rho(\xi, t) d \xi \\
\leq & \left(\gamma_{x}^{2}-\gamma^{2}\right)(q(\eta, t), t),
\end{aligned}
$$


where we used the above claim as regards $\rho(x, t)$. Similarly, if $x \leq q(\eta, t)$, for any $\eta \in\left(x_{0}-\right.$ $\left.\delta, x_{0}+\delta\right)$, we also have

$$
\gamma_{x}^{2}(x, t)-\gamma^{2}(x, t) \leq\left(\gamma_{x}^{2}-\gamma^{2}\right)(q(\eta, t), t) .
$$

This completes the proof of the claim.

For any $x \in\left(x_{0}-\delta, x_{0}+\delta\right)$, by applying our claim to (3.12) and (3.13), we obtain

$$
\begin{aligned}
\frac{d A(q(x, t), t)}{d t} \geq & \frac{1}{2} e^{q(x, t)}\left[u(q(x, t), t)-u_{x}(q(x, t), t)\right]^{2} \\
& +\frac{1}{2} \int_{-\infty}^{q(x, t)} e^{\xi}\left[u(\xi, t)-u_{\xi}(\xi, t)\right]^{2} d \xi>0, \\
\frac{d B(q(x, t), t)}{d t} \leq & -\frac{1}{2} e^{-q(x, t)}\left[u(q(x, t), t)+u_{x}(q(x, t), t)\right]^{2} \\
& -\frac{1}{2} \int_{q(x, t)}^{\infty} e^{-\xi}\left[u(\xi, t)+u_{\xi}(\xi, t)\right]^{2} d \xi<0,
\end{aligned}
$$

which implies $A(q(x, t), t)$ is a strictly increasing function, while $B(q(x, t), t)$ is a strictly decreasing one for a nontrivial solution.

Now we prove Case 1.

From (3.1) and Claim 3, we have

$$
\begin{aligned}
\frac{d}{d t} u_{x}\left(q\left(x_{0}, t\right), t\right)= & \left(u_{x t}+u u_{x x}\right)\left(q\left(x_{0}, t\right), t\right) \\
\leq & \frac{1}{2} u^{2}\left(q\left(x_{0}, t\right), t\right)-\frac{1}{2} u_{x}^{2}\left(q\left(x_{0}, t\right), t\right) \\
& +\frac{1}{2} \gamma^{2}\left(q\left(x_{0}, t\right), t\right)-\frac{1}{2} \gamma_{x}^{2}\left(q\left(x_{0}, t\right), t\right) \\
& -\frac{1}{2} G *\left(\gamma^{2}-\gamma_{x}^{2}\right) \\
\leq & \frac{1}{2} u^{2}\left(q\left(x_{0}, t\right), t\right)-\frac{1}{2} u_{x}^{2}\left(q\left(x_{0}, t\right), t\right) \\
= & \frac{1}{2} A\left(q\left(x_{0}, t\right), t\right) B\left(q\left(x_{0}, t\right), t\right) .
\end{aligned}
$$

Due to the increasing property of $A\left(q\left(x_{0}, t\right), t\right)$ and the decreasing property of $B\left(q\left(x_{0}, t\right), t\right)$ ((3.14) and (3.15)), if we let

$$
m(t):=u_{x}\left(q\left(x_{0}, t\right), t\right)
$$

then

$$
\begin{aligned}
\frac{d}{d t} m(t) & \leq \frac{1}{2} u^{2}\left(q\left(x_{0}, t\right), t\right)-\frac{1}{2} u_{x}^{2}(q(x, t), t) \\
& =\frac{1}{2} A\left(q\left(x_{0}, t\right), t\right) B\left(q\left(x_{0}, t\right), t\right) \\
& \leq \frac{1}{2} A_{0} B_{0}<0 .
\end{aligned}
$$


Suppose the corresponding solution exists globally in time. Since $m(t)$ is strictly decreasing with initial assumption $m(0)<0$, there exists a $t_{1}$ such that for all $t>t_{1}$, we have

$$
m(t)<-\sqrt{E(0)}<0
$$

where

$$
E(0)=\int_{\mathbb{R}} u_{0}^{2}+u_{0 x}^{2}+\gamma_{0}^{2}+\gamma_{0 x}^{2} d x .
$$

Thanks to (3.16) and the following fact:

$$
\|u(x, t)\|_{L^{\infty}(\mathbb{R})}^{2} \leq \frac{1}{2}\|u(x, t)\|_{H^{1}(\mathbb{R})}^{2} \leq \frac{1}{2} E(t)=\frac{1}{2} E(0),
$$

we have the following inequality for $t>t_{0}$ :

$$
\begin{aligned}
\frac{d}{d t} m(t) & \leq-\frac{1}{2} m^{2}(t)+\frac{1}{2} u^{2}\left(q\left(x_{0}, t\right), t\right) \\
& \leq-\frac{1}{2} m^{2}(t)+\frac{1}{4} E(0) \\
& \leq-\frac{1}{4} m^{2}(t) .
\end{aligned}
$$

Then we need the following lemma to finish our proof for Case 1.

Lemma 3.2 [31] Assume that a differentiable function $y(t)$ satisfies

$$
y^{\prime}(t) \leq-C y^{2}(t)+K
$$

with constant $C, K>0$. If we have the initial datum $y(0)=y_{0}<-\sqrt{\frac{K}{C}}$, then the solution goes to $-\infty$ before $t$ tends to $\frac{1}{-C y_{0}+\frac{K}{y_{0}}}$.

Through this lemma, we can see that $m(t)$ goes to $-\infty$ within finite time and Case 1 has been proved.

Now we prove Case 2.

We will prove that after some time Case 2 will change to Case 1 . So it is sufficient to show that there exists a time $T_{0} \in(0, \infty)$, such that $\int_{-\infty}^{q\left(x_{0}, t\right)} e^{\xi} y(\xi, t) d \xi>0$ as $t>T_{0}$.

Suppose not, i.e., $\int_{-\infty}^{q\left(x_{0}, t\right)} e^{\xi} y(\xi, t) d \xi \leq 0$ for any $t>0$. As in Case 2, we have

$$
\int_{-\infty}^{x_{0}} e^{\xi} y_{0}(\xi) d \xi<0, \quad \int_{x_{0}}^{\infty} e^{-\xi} y_{0}(\xi) d \xi=-\epsilon_{0}<0
$$

Then

$$
\lim _{x \rightarrow x_{0}} \int_{x}^{\infty} e^{-\xi} y_{0}(\xi) d \xi=-\epsilon_{0}
$$

which shows that, for $\frac{\epsilon_{0}}{2}>0$, there exists a $\delta^{\prime}>0$, and for any $x \in U\left(x_{0}, \delta^{\prime}\right) \doteq\left\{x|| x-x_{0} \mid<\right.$ $\delta^{\prime}$ \}, we have

$$
\left|\int_{x}^{\infty} e^{-\xi} y_{0}(\xi) d \xi+\epsilon_{0}\right|<\frac{\epsilon_{0}}{2} .
$$




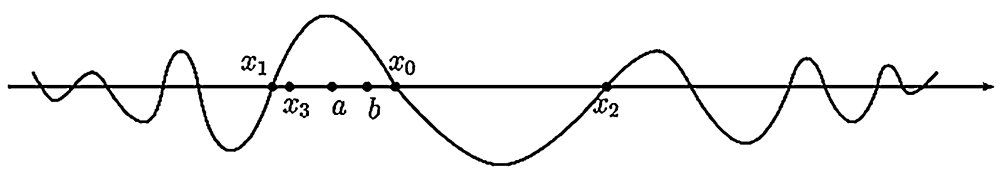

Figure $2 y_{0}$ for Case 2 .

That is to say

$$
\int_{x}^{\infty} e^{-\xi} y_{0}(\xi) d \xi<-\frac{\epsilon_{0}}{2}<0, \quad \text { for any } x \in U\left(x_{0}, \rho\right)
$$

If we set $x_{1}^{*}=\max \left\{x_{1}, x_{0}-\delta\right\}$ and $x_{3}=\max \left\{x_{1}^{*}, x_{0}-\delta^{\prime}\right\}$ (see Figure 2), then

$$
\int_{x_{3}}^{\infty} e^{-\xi} y_{0}(\xi) d \xi<-\frac{\epsilon_{0}}{2}<0
$$

Therefore, for any $x \in\left[x_{3}, x_{0}\right]$,

$$
\int_{-\infty}^{x} e^{\xi} y_{0}(\xi) d \xi<0 \text { and } \int_{x}^{\infty} e^{-\xi} y_{0}(\xi) d \xi<0
$$

When $x \in\left[x_{3}, x_{0}\right] \subseteq\left(x_{0}-\delta, x_{0}+\delta\right)$, we see that $A(q(x, t), t)$ is increasing and $B(q(x, t), t)$ is decreasing, then from the hypothesis we know for all $t>0$

$$
\int_{-\infty}^{q(x, t)} e^{\xi} y(\xi, t) d \xi<\int_{-\infty}^{q\left(x_{0}, t\right)} e^{\xi} y(\xi, t) d \xi \leq 0
$$

and

$$
\int_{q(x, t)}^{\infty} e^{-\xi} y(\xi, t) d \xi<0
$$

Then we obtain

$$
\begin{aligned}
\left(u^{2}-u_{x}^{2}\right)(q(x, t), t) & =\int_{-\infty}^{q(x, t)} e^{\xi} y(\xi, t) d \xi \int_{q(x, t)}^{\infty} e^{-\xi} y(\xi, t) d \xi \\
& >0, \quad \text { for } x \in\left[x_{3}, x_{0}\right]
\end{aligned}
$$

That is to say $\left|u_{x}\right| \leq|u| \leq E(0)$.

Case 3.1 For any $x, y$ satisfying $x<y \in\left[\frac{x_{3}+5 x_{0}}{6}, x_{0}\right]$, there exists a constant $M>0$, such that $0<q(y, t)-q(x, t) \leq M$.

For any $a<b \in\left[\frac{x_{3}+5 x_{0}}{6}, x_{0}\right]$, assume that $b-a=\delta_{0}$. In view of $u(q(x, t), t)<0$ for $x \in$ $\left[x_{3}, x_{0}\right]$, we have

$$
\begin{aligned}
\left(\int_{a}^{b} \sqrt{y_{0}(x)} d x\right)^{2} & \leq \int_{q(a, t)}^{q(b, t)} y(\xi, t) d \xi(q(b, t)-q(a, t)) \\
& \leq-\int_{q(a, t)}^{q(b, t)} u_{\xi \xi} d \xi(q(b, t)-q(a, t))
\end{aligned}
$$




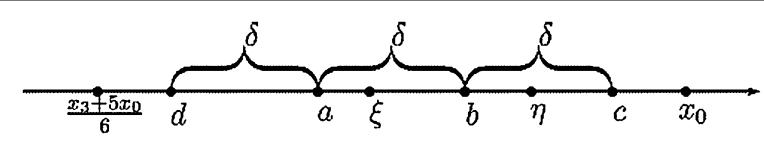

Figure 3 Case 3.1

$$
\begin{aligned}
& =\left[u_{x}(q(a, t), t)-u_{x}(q(b, t), t)\right](q(b, t)-q(a, t)) \\
& \leq M\left[u_{x}(q(a, t), t)-u_{x}(q(b, t), t)\right] .
\end{aligned}
$$

On the other hand, from the boundedness of $u$ and $u_{x}$, it follows that

$$
\left(\int_{a}^{b} \sqrt{y_{0}(x)} d x\right)^{2} \leq 2 E(0)(q(b, t)-q(a, t))
$$

which implies that

$$
q(b, t)-q(a, t) \geq \frac{\left(\int_{a}^{b} \sqrt{y_{0}(x)} d x\right)^{2}}{2 E(0)} .
$$

From the expression of $q(x, t)$, we know that

$$
\frac{d}{d t} \frac{q_{x}(a, t)}{q_{x}(b, t)}=\frac{q_{x}(a, t)}{q_{x}(b, t)}\left[u_{x}(q(a, t), t)-u_{x}(q(b, t), t)\right]
$$

Hence

$$
\int_{0}^{t}\left[u_{x}(q(a, s), s)-u_{x}(q(b, s), s)\right] d s=\ln \frac{q_{x}(a, t)}{q_{x}(b, t)} .
$$

Fix some points $d<a<b<c \in\left[\frac{x_{3}+5 x_{0}}{6}, x_{0}\right]$ (see Figure 3), such that $b-a=a-d=c-b=$ $\delta_{0}>0$, and $\delta_{0}$ is small enough. Because of the convexity of $q(x, t)$, we can deduce that

$$
\begin{aligned}
M & >q(a, t)-q(d, t)>q(b, t)-q(a, t)>q(c, t)-q(b, t) \\
& \geq \frac{\left(\int_{b}^{c} \sqrt{y_{0}(x)} d x\right)^{2}}{2 E(0)} .
\end{aligned}
$$

Therefore, there exist $\eta \in(b, c)$ and $\xi \in(a, b)$, such that

$$
\begin{aligned}
\frac{\left(\int_{b}^{c} \sqrt{y_{0}(x)} d x\right)^{2}}{2 E(0) \delta_{0}} & \leq \frac{q(c, t)-q(b, t)}{c-b}=q_{x}(\eta, t)<q_{x}(b, t)<q_{x}(\xi, t) \\
& =\frac{q(b, t)-q(a, t)}{b-a} \leq \frac{M}{\delta_{0}} .
\end{aligned}
$$

Similarly, we also get

$$
\frac{\left(\int_{a}^{b} \sqrt{y_{0}(x)} d x\right)^{2}}{2 E(0) \delta_{0}}<q_{x}(a, t)<\frac{M}{\delta_{0}}
$$


Combining (3.18) and (3.19), it follows that

$$
\begin{aligned}
\int_{0}^{\infty}\left[u_{x}(q(a, s), s)-u_{x}(q(b, s), s)\right] d s & =\left.\ln \frac{q_{x}(a, t)}{q_{x}(b, t)}\right|_{t=\infty} \\
& <\ln \frac{2 E(0) M}{\left(\int_{b}^{c} \sqrt{y_{0}(x)} d x\right)^{2}}<\infty
\end{aligned}
$$

Then a contradiction is obtained from (3.17): $u_{x}(q(a, t), t)-u_{x}(q(b, t), t)$ is summable with respect to $t$, but $\left(\int_{a}^{b} \sqrt{y_{0}(x)} d x\right)^{2}$ is not.

Case 3.2. There exist some points, say $a^{\prime}<b^{\prime} \in\left[\frac{x_{3}+5 x_{0}}{6}, x_{0}\right]$, such that $q\left(b^{\prime}, t\right)-q\left(a^{\prime}, t\right)>0$ is unbounded.

Different from (3.17), we can deal with the same term as

$$
\begin{aligned}
\left(\int_{a}^{b} \sqrt{y_{0}(x)} d x\right)^{2} & =\left(\int_{q(a, t)}^{q(b, t)} \sqrt{y(\xi)} d \xi\right)^{2} \\
& =\left(\int_{q(a, t)}^{q(b, t)} \sqrt{y(\xi)} \cdot(\xi-q(c, t))^{\frac{1}{2}} \cdot \frac{1}{(\xi-q(c, t))^{\frac{1}{2}}} d \xi\right)^{2} \\
& \leq \int_{q(a, t)}^{q(b, t)} y(\xi)(\xi-q(c, t)) d \xi \cdot \int_{q(a, t)}^{q(b, t)} \frac{1}{\xi-q(c, t)} d \xi \\
& :=J_{1} \cdot J_{2} .
\end{aligned}
$$

According to the convexity of $q(x, t)$, we have

$$
\begin{aligned}
J_{2} & =\ln \frac{q(b, t)-q(c, t)}{q(a, t)-q(c, t)}=\ln \left(\frac{q(b, t)-q(a, t)}{q(a, t)-q(c, t)}+1\right) \\
& <\ln \left(\frac{b-a}{a-c}+1\right)=\ln \frac{b-c}{a-c} .
\end{aligned}
$$

Next we will consider the first term $J_{1}$ in (3.20),

$$
\begin{aligned}
J_{1} & \leq-\int_{q(c, t)}^{q(b, t)} u_{\xi \xi}(\xi-q(c, t)) d \xi \\
& =-\left.u_{\xi}(\xi-q(c, t))\right|_{q(c, t)} ^{q(b, t)}+\int_{q(c, t)}^{q(b, t)} u_{\xi} d \xi \\
& =-u_{x}(q(b, t), t)(q(b, t)-q(c, t))+u(q(b, t), t)-u(q(c, t), t) \\
& <-u_{x}(q(b, t), t)(q(b, t)-q(c, t))-u(q(c, t), t) .
\end{aligned}
$$

From the hypothesis, we know that $q\left(b^{\prime}, t\right)-q\left(a^{\prime}, t\right)$ may reach $\infty$, which means that there exist some times $t_{1}$ and $t_{2}$, such that

$$
\frac{d}{d t}\left(q\left(b^{\prime}, t\right)-q\left(a^{\prime}, t\right)\right)>0, \quad \text { for } t_{1} \leq t \leq t_{2} .
$$

Let $d<c<a<b<a^{\prime}<b^{\prime}$ with $c-d=a-c=b-a=a^{\prime}-b=b^{\prime}-a^{\prime}=\delta_{0}$ (see Figure 4). Thanks to $a^{\prime}<b^{\prime} \in\left[\frac{x_{3}+5 x_{0}}{6}, x_{0}\right]$, we know that the above points all belong to the interval $\left[x_{3}, x_{0}\right]$. First, we prove the following claim. 


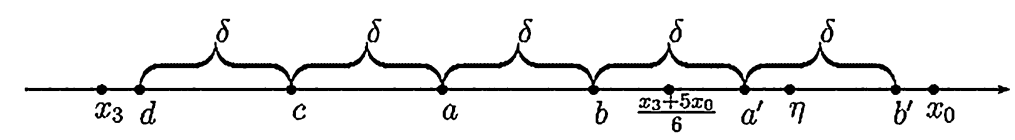

Figure 4 Case 3.2.

Claim 4 For any two adjacent points belong to $\left[x_{3}, a^{\prime}\right]$, say e and $f$ with $e<f$, it satisfies $\frac{d}{d t}(q(f, t)-q(e, t))>0$ as $t_{1} \leq t \leq t_{2}$.

In fact, there exist $\xi \in(q(e, t), q(f, t))$, and $\eta \in\left(q\left(a^{\prime}, t\right), q\left(b^{\prime}, t\right)\right)$, such that

$$
\begin{aligned}
\frac{d}{d t}\left(q\left(b^{\prime}, t\right)-q\left(a^{\prime}, t\right)\right) & =u\left(q\left(b^{\prime}, t\right), t\right)-u\left(q\left(a^{\prime}, t\right), t\right) \\
& =u_{x}(\eta, t)\left(q\left(b^{\prime}, t\right)-q\left(a^{\prime}, t\right)\right), \\
\frac{d}{d t}(q(f, t)-q(e, t)) & =u(q(f, t), t)-u(q(e, t), t) \\
& =u_{x}(\xi, t)(q(f, t)-q(e, t)) .
\end{aligned}
$$

According to $0<u_{x}(\eta, t)<u_{x}(\xi, t)$ and the convexity of $q(x, t)$, we have

$$
\frac{d}{d t}(q(f, t)-q(e, t))>0 \quad \text { for } t \in\left[t_{1}, t_{2}\right]
$$

So the claim is true.

Therefore,

$$
0<\frac{d}{d t}\left(q\left(a^{\prime}, t\right)-q(b, t)\right)=\int_{q(b, t)}^{q\left(a^{\prime}, t\right)} u_{\xi} d \xi \leq u_{x}(q(b, t), t)\left(q\left(a^{\prime}, t\right)-q(b, t)\right),
$$

for all $t \in\left[t_{1}, t_{2}\right]$, which implies

$$
u_{x}(q(b, t), t) \geq \frac{d\left(q\left(a^{\prime}, t\right)-q(b, t)\right)}{d t} \cdot \frac{1}{q\left(a^{\prime}, t\right)-q(b, t)} .
$$

Since $y=u-u_{\xi \xi}>0$,

$$
-u(q(c, t), t)(q(c, t)-q(d, t)) \leq-\int_{q(d, t)}^{q(c, t)} u(\xi) d \xi \leq-\int_{q(d, t)}^{q(c, t)} u_{\xi \xi} d \xi \leq 2 E(0) .
$$

Then

$$
-u(q(c, t), t) \leq \frac{2 E(0)}{q(c, t)-q(d, t)} .
$$

Summarizing these estimates and using the convexity of $q(x, t)$, we can get

$$
\begin{aligned}
J_{1} & <-u_{x}(q(b, t), t)(q(b, t)-q(c, t))-u(q(c, t), t) \\
& \leq-\frac{d\left(q\left(a^{\prime}, t\right)-q(b, t)\right)}{d t} \cdot \frac{1}{q\left(a^{\prime}, t\right)-q(b, t)}(q(b, t)-q(c, t))
\end{aligned}
$$




$$
\begin{aligned}
& +\frac{2 E(0)}{q(c, t)-q(d, t)} \\
\leq & -\frac{d\left(q\left(a^{\prime}, t\right)-q(b, t)\right)}{d t} \cdot \frac{1}{q\left(a^{\prime}, t\right)-q(b, t)}\left(q\left(a^{\prime}, t\right)-q(b, t)\right) \\
& +\frac{2 E(0)}{q\left(a^{\prime}, t\right)-q(b, t)} \\
\leq & -\frac{d\left(q\left(a^{\prime}, t\right)-q(b, t)\right)}{d t}+\frac{2 E(0)}{q\left(a^{\prime}, t\right)-q(b, t)} \\
< & 0,
\end{aligned}
$$

for $q\left(a^{\prime}, t\right)-q(b, t)$ large enough and in the time interval it is increasing.

Putting (3.21) and (3.22) into (3.20), we know that $\left(\int_{a}^{b} \sqrt{y_{0}(x)} d x\right)^{2}$ becomes negative. This is a contradiction.

Therefore, we finish the proof for Case 2.

Finally we finish the proof of our theorem with proving Case 4.

We want to prove that Case 4 can be reduced to the first or the third case, so it is sufficient to prove that there exists a time $T_{0} \in(0, \infty)$, such that $\int_{-\infty}^{q\left(x_{0}, t\right)} e^{\xi} y(\xi, t) d \xi>0$ as $t>T_{0}$.

We suppose that for all $t \in(0, \infty)$, we have $\int_{-\infty}^{q\left(x_{0}, t\right)} e^{x} y(x, t) d x<0$, then get a contradiction. For any $a \in\left[x_{1}^{*}, x_{0}\right]$,

$$
\begin{aligned}
& \left(u_{x}-u\right)(q(a, t), t)=-e^{-q(a, t)} \int_{-\infty}^{q(a, t)} e^{\xi} y(\xi, t) d \xi>0, \\
& \left(u_{x}+u\right)(q(a, t), t)=e^{q(a, t)} \int_{q(a, t)}^{\infty} e^{-\xi} y(\xi, t) d \xi>0 .
\end{aligned}
$$

Summarizing the above two inequalities, we obtain

$$
u_{x}(q(x, t), t) \geq|u(q(x, t), t)|, \quad \text { for } x_{1}^{*}<x<x_{0}, t \geq 0 .
$$

After the above preparation, we have

$$
\begin{aligned}
\left(\int_{a}^{x_{0}} \sqrt{y_{0}(x)} d x\right)^{2} & =\left(\int_{q(a, t)}^{q\left(x_{0}, t\right)} \sqrt{y(q)} d q\right)^{2} \\
& \leq\left(\int_{q(a, t)}^{q\left(x_{0}, t\right)} y(q) e^{q} d q\right)\left(\int_{q(a, t)}^{q\left(x_{0}, t\right)} e^{-q} d q\right) \\
& =\left(\int_{q(a, t)}^{q\left(x_{0}, t\right)}\left(u-u_{x x}\right)(q) e^{q} d q\right)\left(e^{-q(a, t)}-e^{-q\left(x_{0}, t\right)}\right) \\
& \leq\left(u_{x}-u\right)(q(a, t), t)\left(1-e^{q(a, t)-q\left(x_{0}, t\right)}\right) \\
& \leq\left(u_{x}-u\right)(q(a, t), t),
\end{aligned}
$$

which implies

$$
\left(\int_{a}^{x_{0}} \sqrt{y_{0}(x)} d x\right)^{4} \leq\left(u_{x}-u\right)^{2}(q(a, t), t), \quad \text { for } x_{1}^{*}<x<x_{0}, t \geq 0 .
$$


On the other hand, by (3.14), we have

$$
\begin{aligned}
\infty & >-\left.\int_{0}^{\infty} e^{q}\left(u_{x}-u\right)^{2}(q) d t\right|_{x=a} ^{x=x_{0}} \\
& =\int_{0}^{\infty} d t \int_{a}^{x_{0}} e^{q}\left[\left(u_{x}-u\right)^{2}+2\left(u_{x}-u\right) y\right](q) d q \\
& \geq \int_{0}^{\infty} d t \int_{a}^{x_{0}} e^{q} q_{x}\left(u_{x}-u\right)^{2}(q) d x .
\end{aligned}
$$

Since

$$
q+\ln \left(q_{x}\right)=\int_{0}^{t}\left(u_{x}+u\right)(q) d t+x \geq x
$$

which yields

$$
\int_{0}^{\infty} d t \int_{a}^{x_{0}} e^{x}\left(u_{x}-u\right)^{2}(q) d x \leq \int_{0}^{\infty} d t \int_{a}^{x_{0}} e^{q} q_{x}\left(u_{x}-u\right)^{2}(q) d x<\infty
$$

we get

$$
\int_{0}^{\infty}\left(u_{x}-u\right)^{2}(q(x, t), t) d t<\infty, \quad \text { for almost every } x \in\left[x_{1}^{*}, x_{0}\right] .
$$

Then a contradiction is obtained from (3.23): $\left(u_{x}-u\right)^{2}(q)$ taken at $x=a$ is summable with respect to $t$, but $\left(\int_{a}^{x_{0}} \sqrt{y_{0}(x)} d x\right)^{4}$ is not.

So there exists a time $T$, such that when $t>T, \int_{-\infty}^{q\left(x_{0}, t\right)} e^{\xi} y(\xi, t) d \xi>0$. This completes the proof for Case 4.

Remark 3.2 Scrutinizing the proof, we find that the condition of $\rho_{0}(x)$ guarantees that Claim 3 holds. Therefore it can be replaced by

$$
\begin{cases}\rho_{0}(x) \leq 0, & x \in\left(-\infty, x_{0}-\delta\right] \\ \rho_{0}(x) \equiv 0, & x \in\left(x_{0}-\delta, x_{0}+\delta\right) \\ \rho_{0}(x) \geq 0, & x \in\left[x_{0}+\delta, \infty\right)\end{cases}
$$

for all $t>0$. Then the theorem still holds.

Remark 3.3 This blow-up theorem has nothing to do with the initial energy $E(0)$. It is the sign of the initial density $\rho_{0}(x)$ and the sign of $y_{0}(x)$ that play an important role in wave breaking, it is not the size of them that affects it. It is very similar to the necessary and sufficient blow-up condition for the Camassa-Holm equation given by McKean in [20]. 


\section{Author details}

${ }^{1}$ School of Mathematics and Statistics, Tianshui Normal University, Tianshui, 741001, China. ${ }^{2}$ Department of Mathematics, Zhejiang Normal University, Jinhua, 321004, China.

\section{Acknowledgements}

This work is partially supported by ZJNSF (Grant No. LQ13A010008) and NSFC (Grant No. 11226176). Thanks are also given to the anonymous referees for careful reading and suggestions.

\section{Received: 4 November 2013 Accepted: 20 January 2014 Published: 04 Feb 2014}

\section{References}

1. Holm, D, Náraigh, LÓ, Tronci, C: Singular solutions of a modified two-component Camassa-Holm equation. Phys. Rev. E 3(79), 016601 (2009)

2. Guan, C, Karlsen, KH, Yin, Z: Well-posedness and blow-up phenomena for a modified two-component Camassa-Holm equation. In: Nonlinear Partial Differential Equations and Hyperbolic Wave Phenomena. Contemp. Math., vol. 526, pp. 199-220. Am. Math. Soc., Providence (2010)

3. Guo, Z, Zhu, M: Wave breaking for a modified two-component Camassa-Holm system. J. Differ. Equ. 252(3), 2759-2770 (2012)

4. Guo, Z, Zhu, M, Ni, L: Blow-up criteria of solutions to a modified two-component Camassa-Holm system. Nonlinear Anal., Real World Appl. 12, 3531-3540 (2011)

5. Olver, P, Rosenau, P: Tri-Hamiltonian duality between solitons and solitary-wave solutions having compact support. Phys. Rev. E 53, 1900-1906 (1996)

6. Constantin, A, Ivanov, R: On an integrable two-component Camassa-Holm shallow water system. Phys. Lett. A 372 , 7129-7132 (2008)

7. Chen, M, Liu, S, Zhang, Y: A two-component generalization of the Camassa-Holm equation and its solutions. Lett. Math. Phys. 75, 1-15 (2006)

8. Falqui, G: On a Camassa-Holm type equation with two dependent variables. J. Phys. A 39, 327-342 (2006)

9. Escher, J, Lechtenfeld, O, Yin, Z: Well-posedness and blow-up phenomena for the 2-component Camassa-Holm equation. Discrete Contin. Dyn. Syst. 19, 493-513 (2007)

10. Gui, G, Liu, Y: On the global existence and wave-breaking criteria for the two-component Camassa-Holm system. J. Funct. Anal. 258, 4251-4278 (2010)

11. Guo, Z, Zhou, Y: On solutions to a two-component generalized Camassa-Holm equation. Stud. Appl. Math. 124 307-322 (2010)

12. Guo, Z: Blow-up and global solutions to a new integrable model with two components. J. Math. Anal. Appl. 372 , 316-327 (2010)

13. Escher, J, KohImann, M, Lenells, J: The geometry of the two-component Camassa-Holm and Degasperis-Procesi equations. J. Geom. Phys. 61, 436-452 (2011)

14. Holm, D, Ivanov, R: Two component CH system: inverse scattering, peakons and geometry. Inverse Problems 27 , $045013(2011)$

15. Camassa, R, Holm, D: An integrable shallow water equation with peaked solitons. Phys. Rev. Lett. 71, 1661-1664 (1993)

16. Fuchssteiner, B, Fokas, AS: Symplectic structures, their Backlund transformations and hereditary symmetries. Physica D 4(1), $4766(1981 / 1982)$

17. Bressan, A, Constantin, A: Global conservative solutions of the Camassa-Holm equation. Arch. Ration. Mech. Anal. $183,215-239(2007)$

18. Constantin, A, Escher, J: Well-posedness, global existence, and blow-up phenomena for a periodic quasi-linear hyperbolic equation. Commun. Pure Appl. Math. 51, 475-504 (1998)

19. Himonas, A, Misiolek, G, Ponce, G, Zhou, Y: Persistence properties and unique continuation of solutions of the Camassa-Holm equation. Commun. Math. Phys. 271, 511-522 (2007)

20. McKean, HP: Breakdown of a shallow water equation. Asian J. Math. 2, 767-774 (1998)

21. Zhou, Y: Wave breaking for a shallow water equation. Nonlinear Anal. 57, 137-152 (2004)

22. Jiang, Z, Ni, L, Zhou, Y: Wave breaking of the Camassa-Holm equation. J. Nonlinear Sci. 22(2), $235-245$ (2012)

23. Xin, Z, Zhang, P: On the weak solutions to a shallow water equation. Commun. Pure Appl. Math. 53, 1411-1433 (2000)

24. Constantin, A, Strauss, W: Stability of peakons. Commun. Pure Appl. Math. 53, 603-610 (2000)

25. Zhou, Y: Stability of solitary waves for a rod equation. Chaos Solitons Fractals 21, 977-981 (2004)

26. Jiang, Z, Zhou, Y, Zhu, M: Large time behavior for the support of momentum density of the Camassa-Holm equation. J. Math. Phys. 54, 081503 (2013)

27. Mustafa, OG: On smooth traveling waves of an integrable two-component Camassa-Holm shallow water system. Wave Motion 46, 397-402 (2009)

28. Zhang, P, Liu, Y: Stability of solitary waves and wave-breaking phenomena for the two-component Camassa-Holm system. Int. Math. Res. Not. 2010, 1981-2021 (2010)

29. Kato, T: Spectral Theory and Differential Equations. In: Proc. Sympos., Dundee, 1974. Lecture Notes in Math., vol. 48 p. 25. Springer, Berlin (1975), dedicated to Konrad Jorgens

30. Zhou, Y: On solutions to the Holm-Staley b-family of equations. Nonlinearity 23, 369-381 (2010)

31. Zhou, Y: Blow-up of solutions to a nonlinear dispersive rod equation. Calc. Var. Partial Differ. Equ. 25, 63-77 (2006)

10.1186/1029-242X-2014-54

Cite this article as: Ma et al.: Two blow-up criteria of solutions to a modified two-component Camassa-Holm system. Journal of Inequalities and Applications 2014, 2014:54 